\section{RMD Open}

Rheumatic \&

Musculoskeletal Diseases

\title{
Atlas of the OMERACT Heel Enthesitis MRI Scoring System (HEMRIS)
}

Ashish J Mathew (D) , ,2,3 Simon Krabbe (D) , ${ }^{1,2}$ Iris Eshed, ${ }^{4}$ Robert GW Lambert, ${ }^{5,6}$ Jean-Denis Laredo, ${ }^{7}$ Walter P Maksymowych, ${ }^{8,9}$ Frederique Gandjbakhch, ${ }^{10,11}$ Yasser Emad, ${ }^{12}$ Maria Simona Stoenoiu, ${ }^{13,14}$ Violaine Foltz, ${ }^{10,11}$ Paul Bird, ${ }^{15}$ Philippe Carron, ${ }^{16}$ Joel Paschke, ${ }^{8}$ Philip G Conaghan, ${ }^{17,18}$ Susanne J Pedersen, ${ }^{1}$ Daniel Glinatsi, ${ }^{1,19}$ Mikkel Østergaard ${ }^{1,2}$

To cite: Mathew AJ, Krabbe S, Eshed I, et al. Atlas of the OMERACT Heel Enthesitis MRI Scoring System (HEMRIS). RMD Open 2020;6:e001150. doi:10.1136/ rmdopen-2019-001150

Received 15 November 2019 Revised 30 December 2019 Accepted 5 January 2020
Check for updates

\section{(c) Author(s) (or their} employer(s)) 2020. Re-use permitted under CC BY-NC. No commercial re-use. See rights and permissions. Published by BMJ.

For numbered affiliations see end of article.

\section{Correspondence to} Dr Ashish J Mathew; ashishjacobmathew@gmail.com

\section{ABSTRACT}

Objective Assessment of enthesitis, a key feature in spondyloarthritis (SpA) and psoriatic arthritis (PsA), using objective and sensitive methods is pivotal in clinical trials. MRI allows detection of both soft tissue and intra-osseous changes of enthesitis. This article presents an atlas for the Outcome Measures in Rheumatology (OMERACT) Heel Enthesitis Magnetic Resonance ImagingMRI Scoring System (HEMRIS).

Methods Following a preliminary selection of potential examples of each grade, as per HEMRIS definitions, the images along with detailed definitions and reader rules were discussed at web-based, interactive meetings between the members of the OMERACT MRI in Arthritis Working Group.

Results Reference images of each grade of the MRI features to be assessed using HEMRIS, along with reader rules and recommended MRI sequences are depicted.

Conclusion The presented reference images can be used to guide scoring Achilles tendon and plantar fascia (plantar aponeurosis) enthesitis according to the OMERACT HEMRIS in clinical trials and cohorts in which MRI enthesitis is used as an outcome.

\section{INTRODUCTION}

Entheses are sites where tendons, fascia, ligament or joint capsule attach to bones of the appendicular or axial skeleton. ${ }^{2}$ Inflammation at these sites, enthesitis, involves both soft tissue and bone, and is considered as a core pathological process in spondyloarthritis $(\mathrm{SpA})$, including psoriatic arthritis (PsA). ${ }^{34}$ The prevalence of enthesitis in SpA, including PsA, has been reported to be $13.6 \%-35 \%$, with Achilles tendon and plantar fascia insertion being the most common sites. ${ }^{56}$

Assessing enthesitis as primary or secondary outcome in clinical trials requires a comprehensive, sensitive and validated assessment method. Clinical examination using different enthesitis indices has been shown to have low sensitivity and specificity, with suboptimal reproducibility. ${ }^{7}{ }^{8}$ MRI allows

\section{Key messages}

What is already known about this subject?

- The Outcome Measures in Rheumatology (OMERACT) MRI in Inflammatory Arthritis Working Group has published a novel, comprehensive and validated MR scoring system for the heel region (Heel Enthesitis MRI Scoring System (HEMRIS)).

What does this study add?

- The atlas presented in this paper depicts standard reference images for all the grades of different inflammatory and structural pathologies included in the OMERACT HEMRIS, along with line drawings explaining the area to be assessed.

How might this impact on clinical practice?

- This atlas will allow better calibration of readers in future clinical trials with enthesitis at the heel region as an outcome.

- It can also be used as a teaching tool for physicians interested in assessment of enthesitis by MRI.

sensitive assessment of enthesitis, ${ }^{9-11}$ and the OMERACT MRI in Arthritis Working Group has recently developed and validated the OMERACT Heel Enthesitis MRI Scoring System (HEMRIS), along with consensusbased definitions of the MRI features to be scored. ${ }^{12}$ Thus, this method may facilitate a more reliable assessment of enthesitis in clinical trials.

The exact area of assessment and the individual grades of HEMRIS may be difficult for new readers to conceptualise. Furthermore, the applicability and reproducibility of scoring systems have been shown to improve with accessibility of standard reference images for comparison. ${ }^{13-16}$ Therefore, our aim was to develop a reference image atlas for use as a guide to scoring based on the OMERACT HEMRIS, along 
Box 1 Outcome Measures in Rheumatology Heel Enthesitis MRI Scoring System recommendations for MRI acquisition, definitions and scoring of inflammatory and structural pathologies at the entheses

A. Core set of basic MRI sequences and imaging planes

MRI studies that intend to assess inflammatory and structural changes at entheses should include at least the following sequences:

- Short tau inversion recovery (STIR)/T2-weighted fat suppressed (T2wFS) images or, alternatively, gadolinium-enhanced T1-weighted fat suppressed images.

- T1-weighted images without gadolinium enhancement (not mandatory if only inflammation is being assessed).

Suggested imaging planes:

- Achilles tendon: sagittal and preferably also axial.

- Plantar fascia: sagittal and preferably also coronal.

\section{B. Definitions and grades of inflammatory and structural pathologies at the Achilles tendon insertion to calcaneum}

\section{Intratendon hypersignal (STIR/T2wFS)}

\section{Definition}

Signal characteristics consistent with increased water content/inflammation within the tendon, close to its insertion.

Grades

0 : No intratendon hypersignal.*

1: Minimal intratendon hypersignal spots ${ }^{*}(\leq 25 \%$ of the tendon volume).

2: Moderate intratendon hypersignal ${ }^{\star}(>25 \%$ and $\leq 50 \%$ of the tendon volume).

3: Severe intratendon hypersignal ${ }^{*}(>50 \%$ of the tendon volume).

*Intratendon hypersignal should be assessed from the tendon insertion up to $2 \mathrm{~cm}$ proximal to the posterosuperior corner of calcaneum (marked in the line drawing) on all the available images.

\section{Peritendon hypersignal (STIR/T2wFS) \\ Definition}

Signal characteristics consistent with increased water content/inflammation in the soft tissues surrounding the tendon, close to its insertion.

Grades

0 : No hypersignal.*

1: Mild focal hypersignal.*

2: Moderate† hypersignal. ${ }^{*}$

3: Severe† hypersignal. ${ }^{*}$

†By comparison with reference images.

*From tendon insertion up to $2 \mathrm{~cm}$ proximal to the posterosuperior corner of calcaneum (marked in the line drawing).

\section{Bone marrow oedema}

\section{Definition}

Bone marrow oedema (BME) should be assessed in the bone from the entheseal insertion to a depth of $1 \mathrm{~cm}$ on all available images.

\section{Grades}

The scale is $0-3$, based on the proportion of bone with oedema, compared with the 'assessed bone volume', judged on all available images.

0 : No oedema.

$1: 1 \%-33 \%$ of the bone is edematous (ie, BME occupying $1 \%-33 \%$ of the assessed bone volume).

2: $34 \%-66 \%$ of the bone is edematous.

3: $67 \%-100 \%$ of the bone is edematous.

If the lesion is judged borderline, ie, 1 vs 2 or 2 vs 3 , lesion intensity may be considered. For example, if a lesion is borderline between 1 (mild) and 2 (moderate), it may be scored 1 (mild) if not judged intense. Similarly, if a lesion is borderline between 2 (moderate) and 3 (severe), it may be scored 3 (severe) if judged intense.

\section{Retrocalcaneal bursitis}

\section{Definition}

Signal characteristics consistent with increased water content/inflammation in an above-normal sized bursa.

Grades

0 : No hypersignal or maximal diameter of hypersignal in the shorter of two perpendicular dimensions to be $<0.25 \mathrm{~cm}$.

1: Maximal diameter of hypersignal in the shorter of two perpendicular dimensions to be $\geq 0.25 \mathrm{~cm}$ to $<0.5 \mathrm{~cm}$.

2: Maximal diameter of hypersignal in the shorter of two perpendicular dimensions to be $\geq 0.5 \mathrm{~cm}$ to $<1.0 \mathrm{~cm}$.

3: Maximal diameter of hypersignal in the shorter of two perpendicular dimensions to be $\geq 1.0 \mathrm{~cm}$.

\section{Tendon thickening}

Definition

Abnormal thickening of the tendon close to its insertion. ${ }^{*}$

Grades

0 : None. 


\section{Box 1 Continued}

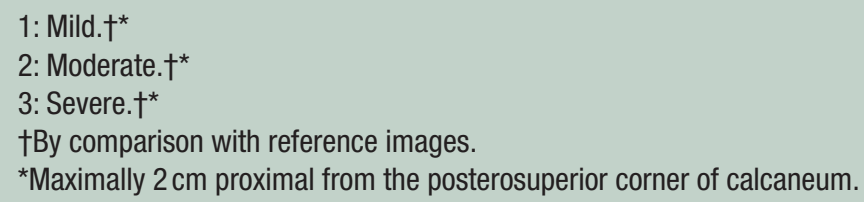

6. Calcaneal enthesophyte (Achilles tendon)

Definition

Abnormal bone formation at the insertion of tendon into the bone.

Grades

0 : None.

1: Small.*

2: Medium-sized. *

3: Large. ${ }^{*}$

*By comparison with reference images.

\section{Calcaneal bone erosion (Achilles tendon)}

Definition

A sharply marginated bone lesion, with typical signal characteristics and a visible cortical break, located close to the tendon insertion.

Grades

0 : None.

1: Small.*

2: Medium-sized. *

3: Large.*

${ }^{*}$ By comparison with reference images.

C. Definitions and grades of inflammatory and structural pathologies at the plantar fascia insertion to calcaneum

1. Intrafascia hypersignal (STIR/T2wFS)

Definition

Signal characteristics consistent with increased water content/inflammation within the fascia, close to its insertion.

Grades

0 : No intrafascia hypersignal. *

1: Minimal intrafascia hypersignal ${ }^{*}(\leq 25 \%$ of the fascia volume $)$.

2: Moderate intrafascia hypersignal ${ }^{\star}(>25 \%$ and $\leq 50 \%$ of the fascia volume).

3: Severe intrafascia hypersignal* $(>50 \%$ of the fascia volume).

*Intratendon hypersignal should be assessed from the fascia insertion up to $2 \mathrm{~cm}$ proximal to the anterior margin of the plantar tuberosity (marked in the line drawing) on all the available images.

\section{Perifascia hypersignal (STIR/T2wFS)}

\section{Definition}

Signal characteristics consistent with increased water content/inflammation in the soft tissues surrounding the fascia, close to its insertion.

\section{Grades}

0 : No hypersignal.*

1: Mild focal hypersignal.*

2: Moderate† hypersignal.*

3: Severe† hypersignal. ${ }^{*}$

†By comparison with reference images.

*From fascia insertion up to $2 \mathrm{~cm}$ proximal to the anterior margin of the plantar tuberosity (marked in the line drawing).

\section{Bone marrow oedema}

\section{Definition}

Bone marrow oedema should be assessed in the bone from the fascia insertion to a depth of $1 \mathrm{~cm}$ on all available images.

Grades

The scale is $0-3$, based on the proportion of bone with oedema, compared with the 'assessed bone volume', judged on all available images.

0 : No oedema.

$1: 1 \%-33 \%$ of the bone is edematous (ie, BME occupying $1 \%-33 \%$ of the assessed bone volume).

2: $34 \%-66 \%$ of the bone is edematous.

$3: 67 \%-100 \%$ of the bone is edematous.

Continued 


\section{Box 1 Continued}

If the lesion is judged borderline, ie, 1 vs 2 or 2 vs 3 , lesion intensity may be considered. For example, if a lesion is borderline between 1 (mild) and 2 (moderate), it may be scored 1 (mild) if not judged intense. Similarly, if a lesion is borderline between 2 (moderate) and 3 (severe), it may be scored 3 (severe) if judged intense.

\section{Fascia thickening \\ Definition}

Abnormal thickening of the fascia close to its insertion.

Grades

0 : None.

1: Mild. $\dagger^{*}$

2: Moderate. $\dagger^{*}$

3: Severe. $\dagger^{\star}$

†By comparison with reference images.

*Maximally $2 \mathrm{~cm}$ proximal to the anterior margin of the plantar tuberosity.

\section{Calcaneal enthesophyte (plantar fascia) \\ Definition}

Abnormal bone formation at the insertion of fascia into the bone.

Grades

0 : None.

1: Small.*

2: Medium-sized.*

3: Large.*

*By comparison with reference images.

\section{Calcaneal bone erosion (plantar fascia) \\ Definition}

A sharply marginated bone lesion, with typical signal characteristics and a visible cortical break, located close to the fascia insertion.

Grades

0 : None.

1: Small.*

2: Medium-sized.*

3: Large*

*By comparison with reference images.

with presenting an updated list of definitions and reader rules.

\section{METHODS}

Images representing each MRI feature, as per HEMRIS definitions, were collected from working group members and a preliminary selection of potential examples of each grade were selected by two group members experienced in the HEMRIS method, and presented for general discussion at three web-based, interactive meetings between the members (rheumatologists and radiologists) of the OMERACT MRI in Arthritis Working Group. At these web-based meetings example images of each grade of MRI feature were discussed, as were detailed definitions and reader rules, and consensus on the final selection was reached. All participating members approved the final definitions, reader rules and image selection.

\section{RESULTS}

The MRI features to be scored in HEMRIS, along with an updated set of their definitions, recommended MRI sequences and reader rules are described in box 1 . Representative examples of different grades for each of the MRI features to be assessed in HEMRIS are presented in figures 1-4. A line drawing, depicting the area of focus while scoring each MRI feature according to HEMRIS, is included.

\section{DISCUSSION}

HEMRIS is the first international consensus-based and validated, comprehensive MRI scoring system for peripheral enthesitis in patients with SpA. In this atlas, we have depicted different grades of each MRI pathologies to be scored using HEMRIS for ease of scoring enthesitis in the heel region in patients with $\mathrm{SpA} / \mathrm{PsA}$ in clinical trials or cohorts.

For efficient utility of this atlas, the reader should be adept to the relevant anatomy of the region, the MRI appearance of Achilles tendon and plantar fascia (plantar aponeurosis) entheses using short tau inversion recovery and T1-weighted sequences in a Digital Imaging and Communications in Medicine viewer, and common pitfalls in assessment. Before assigning a score, the reader is recommended 
Line drawing
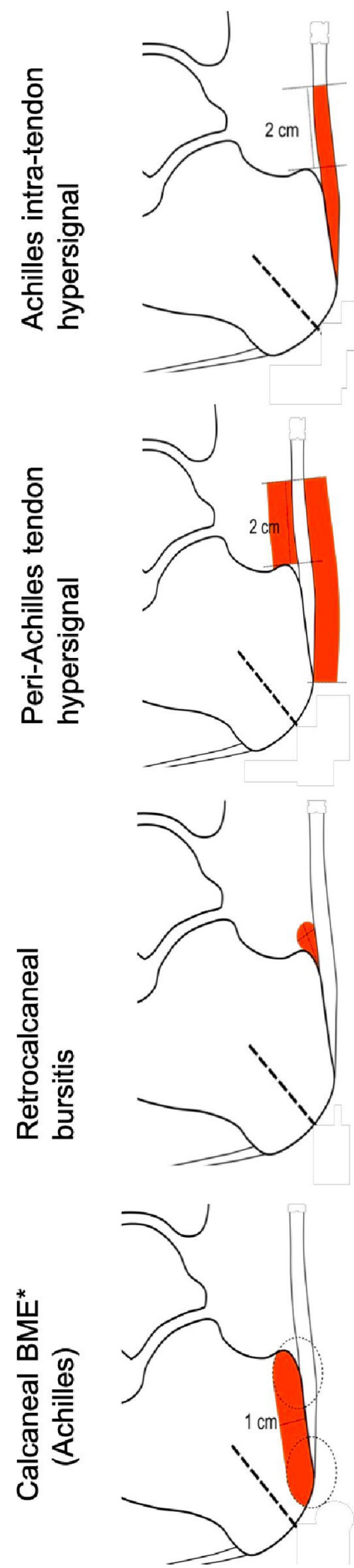

Grade 0
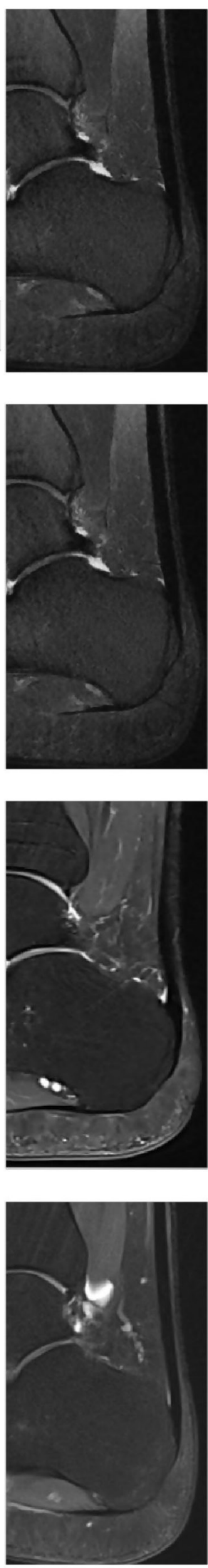

Grade 1
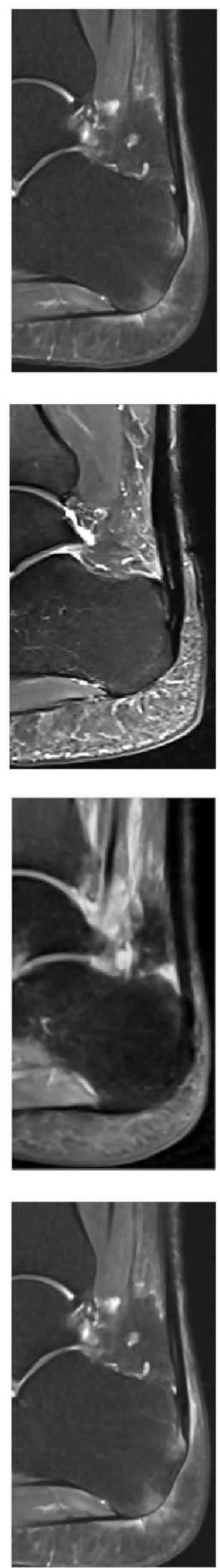

Grade 2
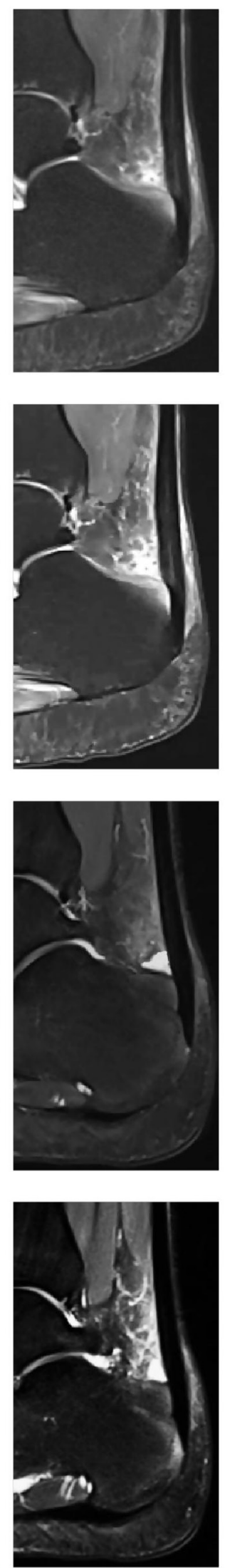

Grade 3
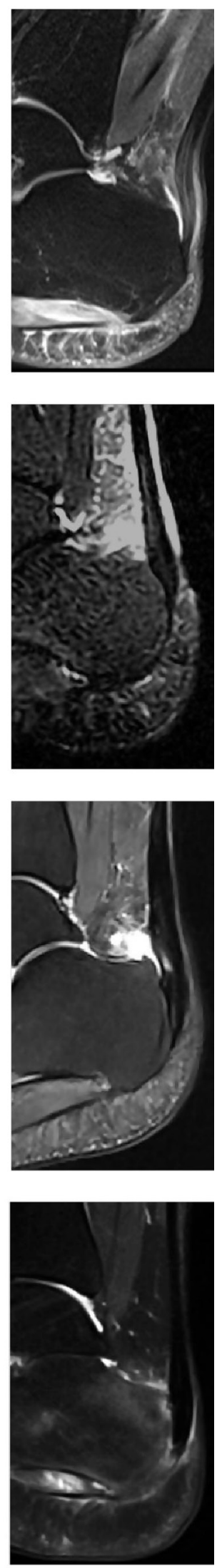

Figure 1 Heel Enthesitis MRI Scoring System grades for inflammatory pathologies at the Achilles tendon enthesis, illustrated by sagittal short tau inversion recovery MR images. A line drawing (left) depicts the area to assess. *BME, bone marrow oedema. 

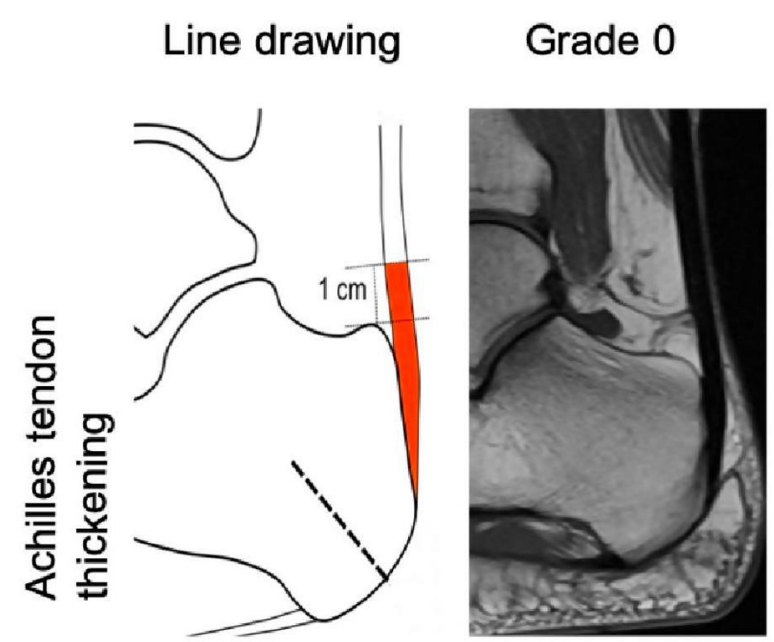

Grade 1

Grade 2

Grade 3
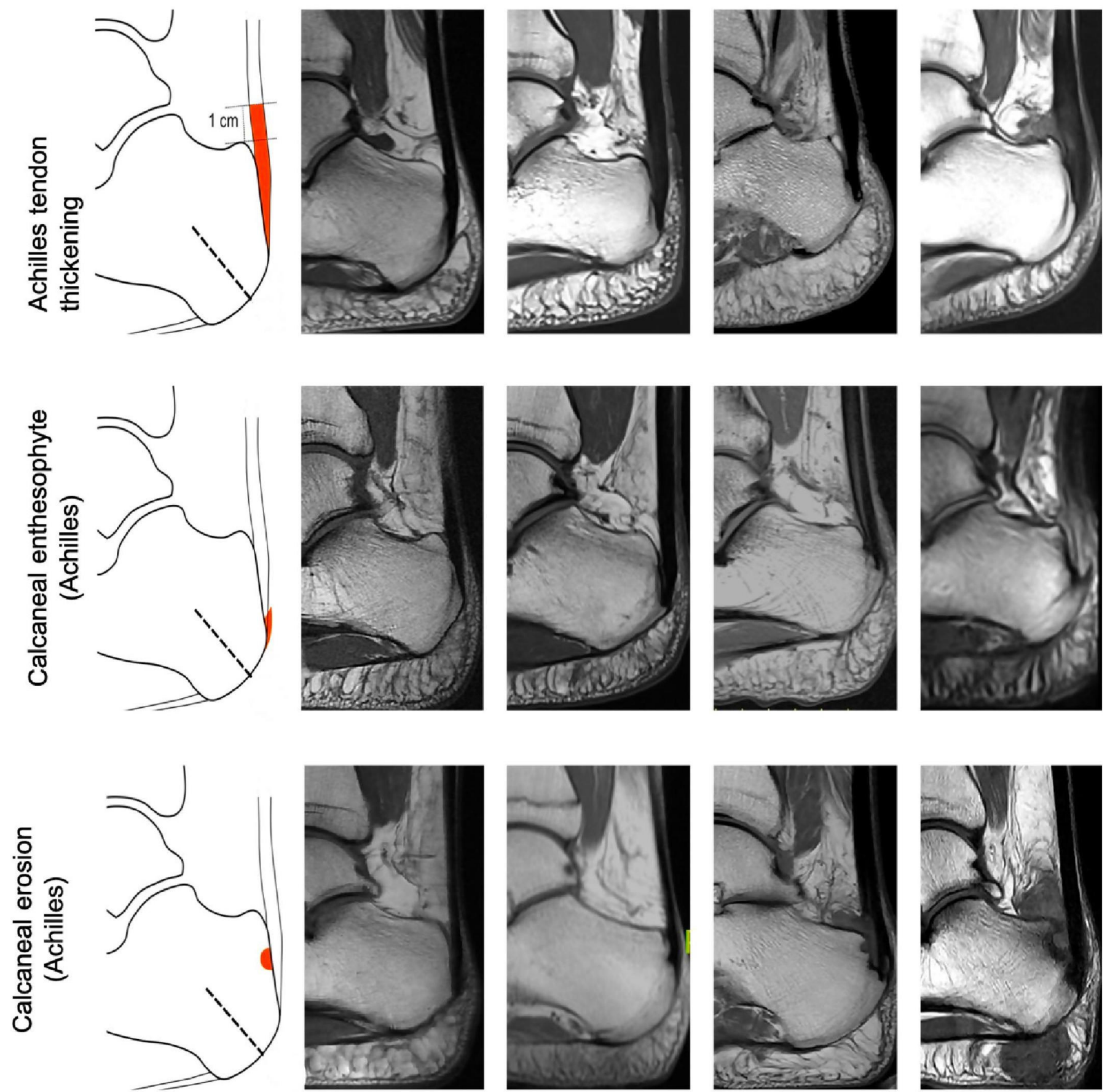

Figure 2 Heel Enthesitis MRI Scoring System grades for structural pathologies at the Achilles tendon enthesis, illustrated by sagittal T1-weighted MR images. A line drawing (left) depicts the area to assess. 

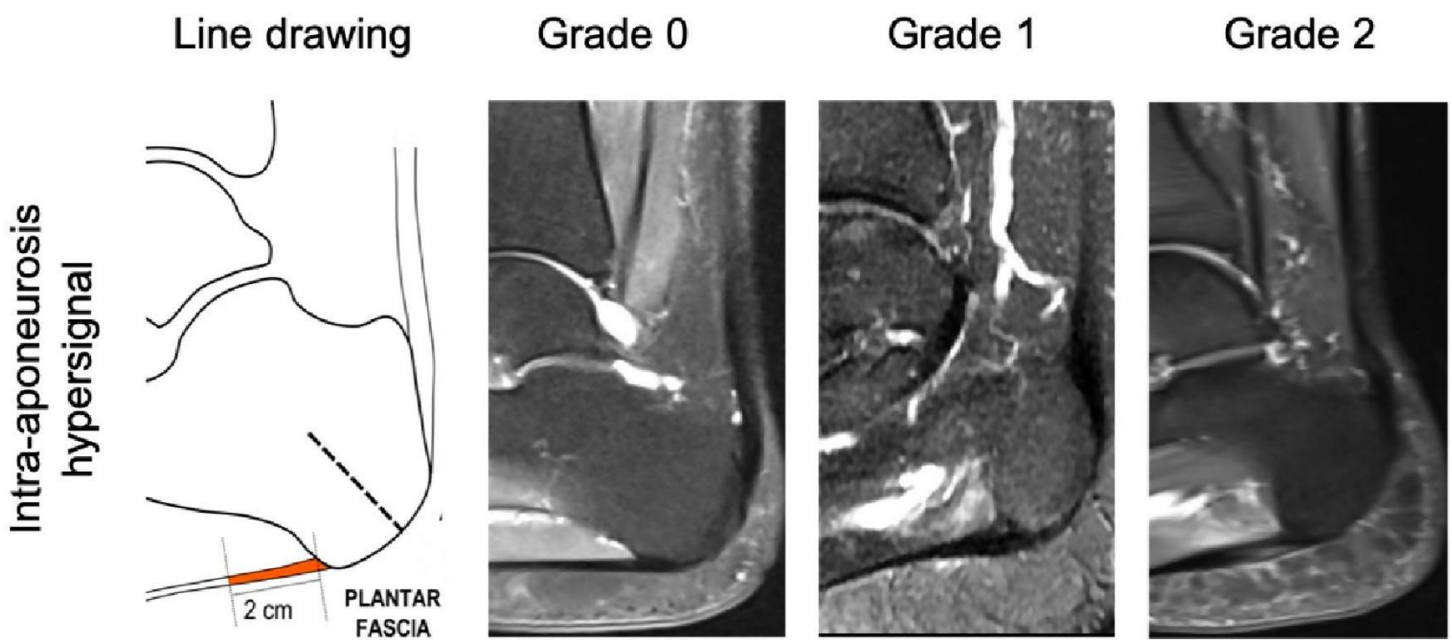

Grade 3
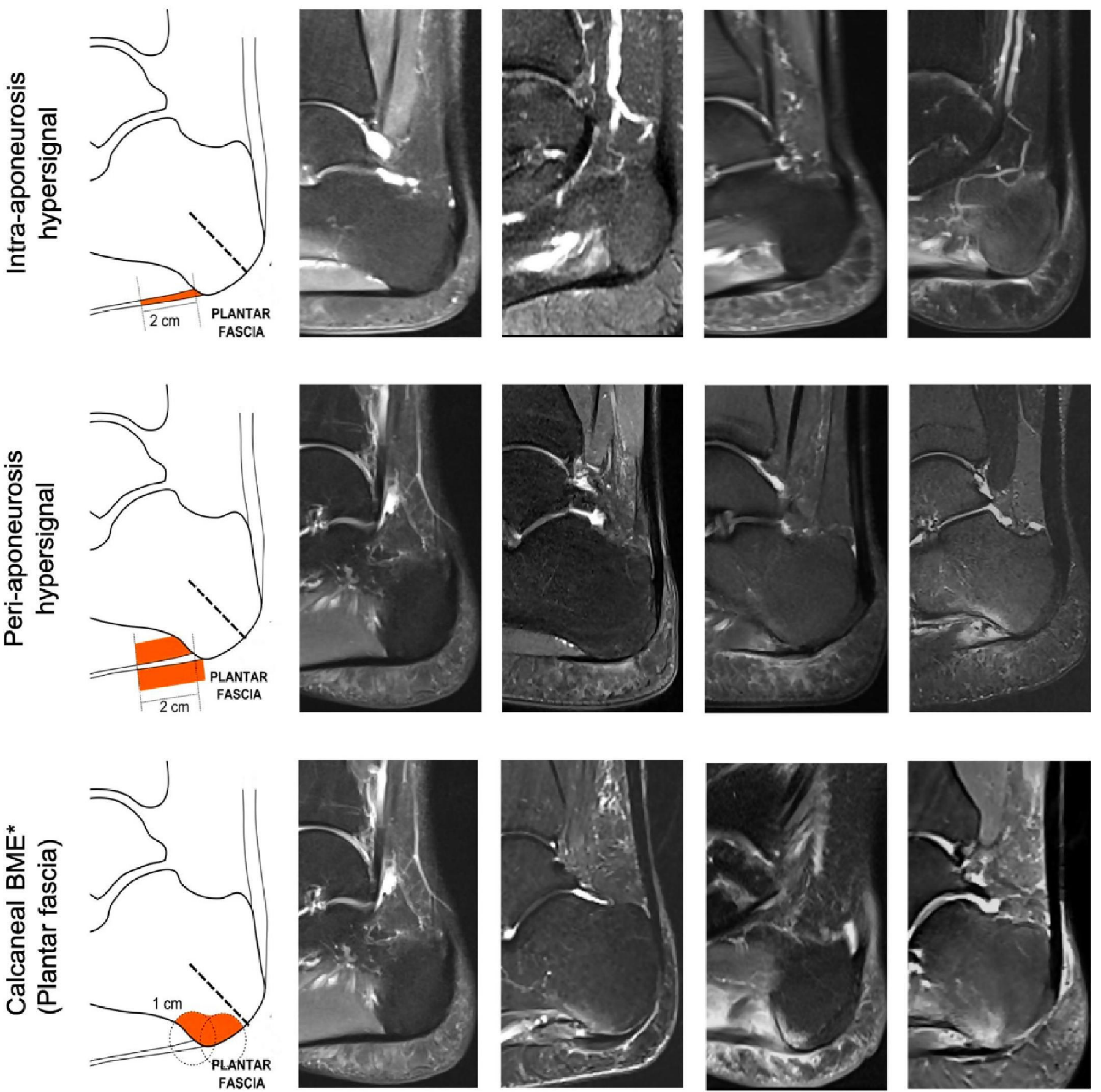

Figure 3 Heel Enthesitis MRI Scoring System grades for inflammatory pathologies at the plantar fascia enthesis, illustrated by sagittal short tau inversion recovery MR images. A line drawing (left) depicts the area to assess. *BME, bone marrow edema. 
Line drawing
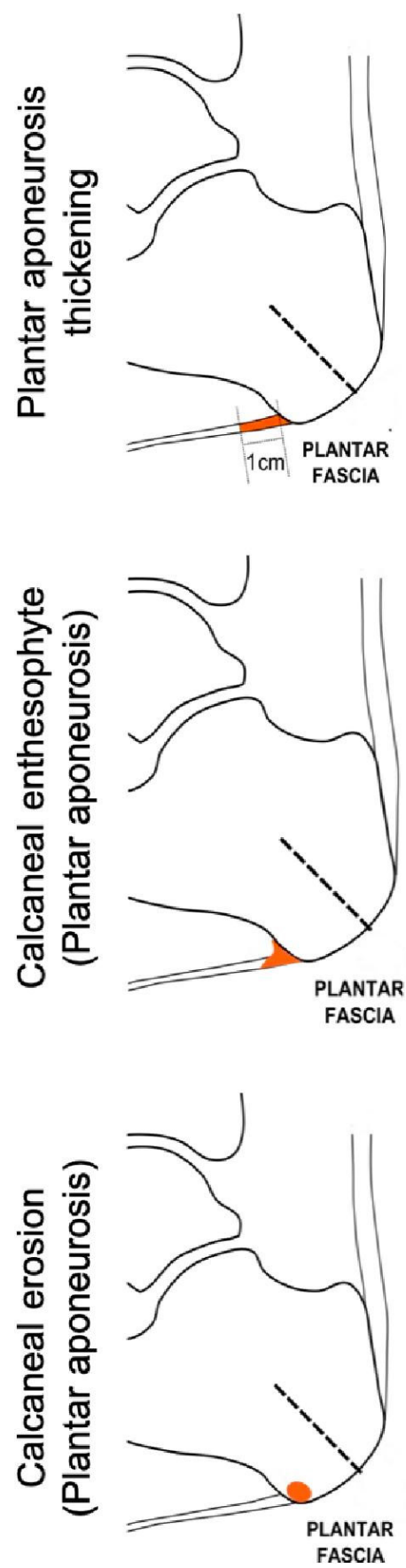

Grade 0
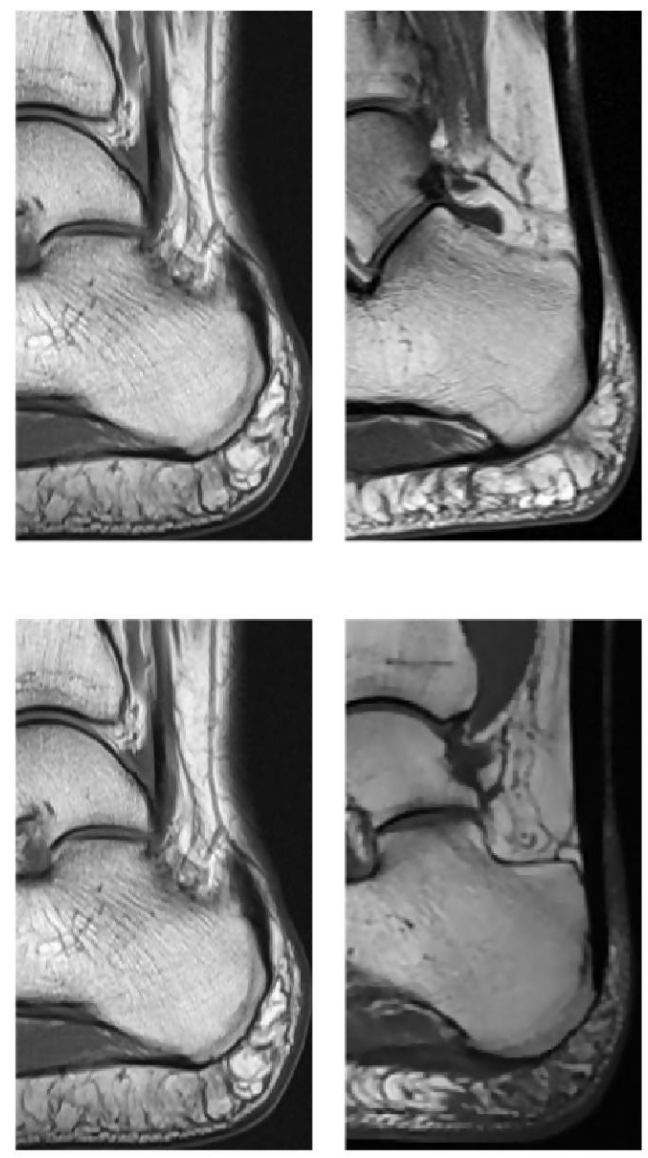

Grade 2
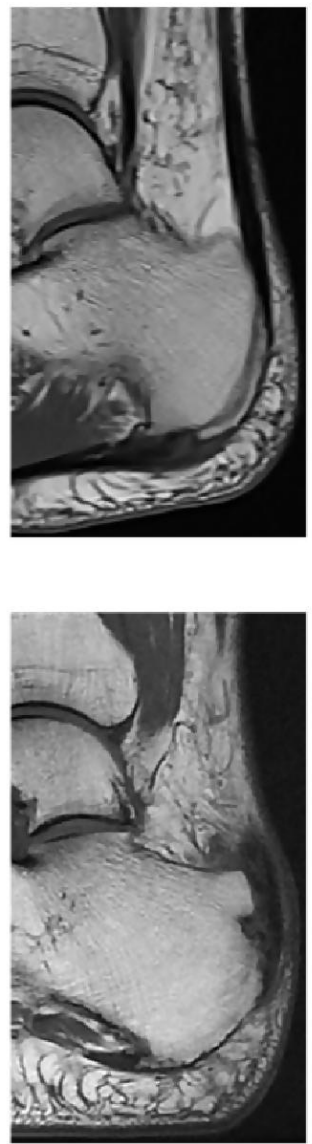

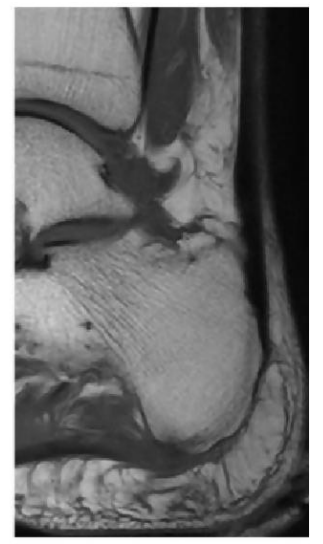

Grade 3

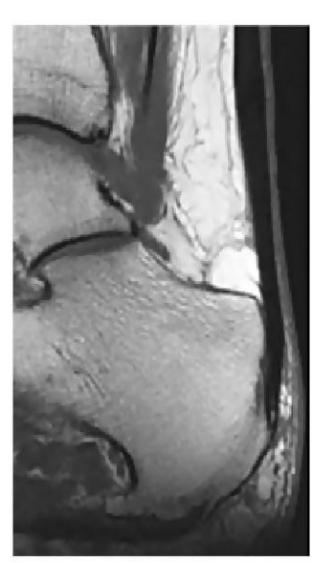

Figure 4 Heel Enthesitis MRI Scoring System grades for structural pathologies at the plantar fascia enthesis, illustrated by sagittal T1-weighted MR images. A line drawing (left) depicts the area to assess.

to window each image appropriately in order to prevent overappreciation or underappreciation of inflammation, and to scroll through all the available images, comparing them with the reference images and grade definitions in the present atlas. Interpretation of MRI finding if using only one imaging plane should be done with caution, especially for small lesions like erosions. For scoring structural lesions it is advisable to have two planes. Fat suppression is sometimes inhomogeneous across an MRI image. One should avoid over-reading inflammation due to hyperintensity resulting from improper fat suppression. Calibration with a trained HEMRIS reader is highly recommended to provide reliable assessments and enhance the overall outcome. ${ }^{1317}$ Although the exact reading time has not been measured, a calibrated reader should generally be able to complete scoring of one heel region in less than 15 minutes.

Sum scores of inflammatory lesions (intratendon hypersignal, peritendon hypersignal, bone marrow oedema, retrocalcaneal bursitis) and structural lesions (tendon thickening, calcaneal enthesophyte, calcaneal bone erosion) can be calculated by summing up the individual pathology scores at Achilles tendon and plantar 
fascia entheses. For Achilles tendon and plantar fascia entheses, the possible range of the HEMRIS inflammation sum score will be $0-12$ and $0-9$, respectively, and the corresponding range of the HEMRIS structural sum scores will be $0-9$, respectively.

In conclusion, we have provided an easy-to-use set of standard reference images for the HEMRIS method to improve calibration between readers in clinical trials and cohorts. This may also be used as a handy teaching tool for new readers interested in MRI assessment of enthesitis at the heel region.

\section{Author affiliations}

${ }^{1}$ Copenhagen Center for Arthritis Research, Center for Rheumatology and Spine Diseases, Copenhagen University Hospital, Glostrup, Denmark

${ }^{2}$ Department of Clinical Medicine, Faculty of Health and Medical Sciences, University of Copenhagen, Copenhagen, Denmark

${ }^{3}$ Clinical Immunology and Rheumatology, Christian Medical College, Vellore, India

${ }^{4}$ Department of Diagnostic Imaging, Sheba Medical Center, Affiliated to the Sackler School of Medicine, Tel Aviv University, Tel Aviv, Israel

${ }^{5}$ Department of Radiology and Diagnostic Imaging, University of Alberta, Edmonton, Alberta, Canada

${ }^{6}$ Medical Imaging Consultants, Edmonton, Alberta, Canada

${ }^{7}$ Service de Radiologie, Hôpital Lariboisiére, APHP \& Université Paris-Diderot, Paris, France

${ }^{8}$ CaRE (Canadian Research Education) Arthritis, Edmonton, Alberta, Canada

${ }^{9}$ Department of Medicine, University of Alberta, Edmonton, Alberta, Canada

${ }^{10}$ Department of Rheumatology, CHU Pitié-Salpêtrière, Assistance PubliqueHôpitaux de Paris, Paris, France

${ }^{11}$ Paris 6 University, GRC-UPMC 08, Pierre Louis Institute of Epidemiology and

Public Health, Paris, France

${ }^{12}$ Rheumatology, Faculty of Medicine, Cairo University, Giza, Egypt

${ }^{13}$ Rheumatology, Cliniques Universitaires Saint-Luc, Brussels, Belgium

${ }^{14}$ Institut de Recherche Experimentale et Clinique, Universite Catholique de Louvain La Faculte de Medecine, Brussels, Belgium

${ }^{15}$ Division of Medicine, University of New South Wales, Sydney, New South Wales, Australia

${ }^{16}$ Rheumatology, Ghent University Hospital, Ghent, Belgium

${ }^{17}$ Leeds Institute of Rheumatic and Musculoskeletal Medicine, University of Leeds, Leeds, UK

${ }^{18}$ Leeds Teaching Hospitals NHS Trust, NIHR Leeds Biomedical Research Centre, Leeds, UK

${ }^{19}$ Rheumatology, Skaraborg Hospital Skövde, Skovde, Sweden

Contributors All the authors contributed equally towards the work involved and manuscript preparation.

Funding PGC is supported in part by the UK National Institute for Health Research (NIHR) Leeds Biomedical Research Centre.

Disclaimer The views expressed are those of the author(s) and not necessarily those of the NHS, the NIHR or the Department of Health.

Competing interests None declared.

Patient consent for publication Not required.

Provenance and peer review Not commissioned; externally peer reviewed.

Data availability statement All data relevant to the study are included in the article or uploaded as supplementary information. All the images used are included in this atlas.

Open access This is an open access article distributed in accordance with the Creative Commons Attribution Non Commercial (CC BY-NC 4.0) license, which permits others to distribute, remix, adapt, build upon this work non-commercially, and license their derivative works on different terms, provided the original work is properly cited, appropriate credit is given, any changes made indicated, and the use is non-commercial. See: http://creativecommons.org/licenses/by-nc/4.0/.

ORCID iDs

Ashish J Mathew http://orcid.org/0000-0002-2061-2042

Simon Krabbe http://orcid.org/0000-0002-2877-1582

\section{REFERENCES}

1 McGonagle D, Gibbon W, Emery P. Classification of inflammatory arthritis by enthesitis. Lancet 1998;352:1137-40.

2 Benjamin M, Moriggl B, Brenner E, et al. The "enthesis organ" concept: why enthesopathies may not present as focal insertional disorders. Arthritis Rheum 2004;50:3306-13.

3 Benjamin M, McGONAGLE D. The anatomical basis for disease localisation in seronegative spondyloarthropathy at entheses and related sites. J Anat 2001;199:503-26.

4 McGonagle D, Marzo-Ortega H, O'Connor P, et al. Histological assessment of the early enthesitis lesion in spondyloarthropathy. Ann Rheum Dis 2002;61:534-7.

5 de Winter JJ, van Mens LJ, van der Heijde D, et al. Prevalence of peripheral and extra-articular disease in ankylosing spondylitis versus non-radiographic axial spondyloarthritis: a meta-analysis. Arthritis Res Ther 2016;18:196.

6 Polachek A, Li S, Chandran V, et al. Clinical enthesitis in a prospective longitudinal psoriatic arthritis cohort: incidence, prevalence, characteristics, and outcome. Arthritis Care Res 2017;69:1685-91.

7 Eshed I, Bollow M, McGonagle DG, et al. Mri of enthesitis of the appendicular skeleton in spondyloarthritis. Ann Rheum Dis 2007:66:1553-9.

8 Marchesoni A, Atzeni F, Spadaro A, et al. Identification of the clinical features distinguishing psoriatic arthritis and fibromyalgia. $J$ Rheumatol 2012;39:849-55.

9 Mathew AJ, Krabbe S, Kirubakaran R, et al. Utility of magnetic resonance imaging in diagnosis and monitoring Enthesitis in patients with spondyloarthritis: an OMERACT systematic literature review. $J$ Rheumatol 2019;46:1207-14.

10 McGonagle D, Gibbon W, O'Connor P, et al. Characteristic magnetic resonance imaging entheseal changes of knee synovitis in spondylarthropathy. Arthritis Rheum 1998;41:694-700.

11 Lambert RGW, Dhillon SS, Jhangri GS, et al. High prevalence of symptomatic enthesopathy of the shoulder in ankylosing spondylitis: deltoid origin involvement constitutes a hallmark of disease. Arthritis Rheum 2004;51:681-90.

12 Mathew AJ, Krabbe S, Eshed I, et al. The OMERACT MRI in enthesitis initiative: definitions of key pathologies, suggested MR sequences, and a novel heel enthesitis scoring system. J Rheumatol 2019;46:1232-8.

13 Maksymowych WP, Dhillon SS, Chiowchanwisawakit P, et al. Development and validation of web-based training modules for systematic evaluation of active inflammatory lesions in the spine and sacroiliac joints in spondyloarthritis. J Rheumatol Suppl 2009;84:48-57.

14 Conaghan P, Bird P, Ejbjerg B, et al. The EULAR-OMERACT rheumatoid arthritis MRI reference image atlas: the metacarpophalangeal joints. Ann Rheum Dis 2005;64 Suppl 1:i11-21.

15 Ejbjerg B, McQueen F, Lassere M, et al. The EULAR-OMERACT rheumatoid arthritis MRI reference image atlas: the wrist joint. Ann Rheum Dis 2005;64 Suppl 1:i23-47.

16 Kroon FPB, Peterfy CG, Conaghan PG, et al. Atlas for the OMERACT thumb base osteoarthritis MRI scoring system (TOMS). RMD Open 2018;4:e000583.

17 Bird P, Joshua F, Lassere M, et al. Training and calibration improve inter-reader reliability of joint damage assessment using magnetic resonance image scoring and computerized erosion volume measurement. J Rheumatol 2005;32:1452-8. 